Elsevier required licence: (c) <2019>.

This manuscript version is made available under the CC-BY-NC-ND 4.0 license

http://creativecommons.org/licenses/by-nc-nd/4.0/

The definitive publisher version is available online at

https://www.sciencedirect.com/science/article/pii/S0168192319300115?via\%3Dihub 


\section{Risk assessment using transfer learning for grassland fires}

Xing-peng Liu ${ }^{a, b, c}$, Guang-quan Zhang ${ }^{d, *}$, Jie Lu ${ }^{d}$, Ji-quan Zhang ${ }^{a, b, c, ~ *}$

a School of Environment, Northeast Normal University, Changchun 130024, P. R. China

${ }^{\mathrm{b}}$ Key Laboratory for Vegetation Ecology, Ministry of Education, Changchun 130024, P. R. China

${ }^{\mathrm{c}}$ State Environmental Protection Key Laboratory of Wetland Ecology and Vegetation Restoration,

Northeast Normal University, Changchun, 130024, P. R. China

${ }^{\mathrm{d}}$ Decision Systems \& e-Service Intelligence Lab, Centre for Artificial Intelligence, Faculty of

Engineering and Information Technology, University of Technology Sydney, PO Box 123,

Broadway, NSW 2007, Australia

${ }^{*}$ Corresponding author. Email: guangquan.zhang@ust.edu.au (G. Q. Zhang); zhangjq022@nenu.edu.cn

(J. Q. Zhang) 
Abstract: A new direction of risk assessment research in grassland fire management is data-driven prediction, in which data are collected from particular regions. Since some regions have rich datasets that can easily generate knowledge for risk prediction, and some have no data available, this study addresses how we can leverage the knowledge learned from one grassland risk assessment to assist with a current assessment task. In this paper, we first introduce the transfer learning methodology to map and update risk maps in grassland fire management, and we propose a new grassland fire risk analysis method. In this study, two major grassland areas (Xilingol and Hulunbuir) in northern China are selected as the study areas, and five representative indicators (features) are extracted from grassland fuel, fire climate, accessibility, human and social economy. Taking Xilingol as the source domain (where sufficient labelled data are available) and Hulunbuir as the target domain (which contains insufficient data but requires risk assessment/prediction), we then establish the mapping relationship between grassland fire indicators and the degrees of grassland fire risk by using a transfer learning method. Finally, the fire risk in the Hulunbuir grassland is assessed using the transfer learning method. Experiments show that the prediction accuracy reached $87.5 \%$ by using the transfer learning method, representing a significant increase over existing methods.

Keywords: Risk assessment; transfer learning; fire climate; grassland fire

\section{Introduction}


destructive; it is one of the most serious natural disasters occurring in grasslands (Cheney et al. 1998; Liu et al. 2015; Cao et al. 2015). Fire burns vegetation, livestock, and important species, destroying pastures and soil and causing dust storms and soil erosion (Kandya et al. 1998). In the context of global climate change, the impact of wildfires will increase with the increasing frequency of extreme climate events (Liu \& Stanturf et al. 2010). Therefore, it is necessary to assess wildfire risk across an entire area to support grassland management. The assessment of fire risk, as defined by Bachmann and Allgower (2001), requires assessing the possibility of future occurrences of fires and potential losses.

Fire risk assessment is the decision-making basis for fire managers. Therefore, many researchers have given attention to grassland fire risk in recent years (Zhang et al. 2006; Zhang et al. 2010; Ager et al. 2011; Chuvieco et al. 2014; Thompson et al. 2015; Zhang et al. 2015). Risk analysis is the key point of research on grassland fire risk management. Because risk analysis involves several factors of grassland fire behaviour and the social environment and because the relationships between those factors are complex, the formation mechanism of grassland fire risk is not clear, which causes the evaluation accuracy of grassland fire risk to be low. In actual applications, the multiple factors used in the assessment are difficult to obtain; thus, grassland fire risk assessment is time-consuming and labour-intensive. Furthermore, in many remote areas, fire risk is often more difficult to assess because of a lack of grassland fire data. Therefore, grassland fire risk assessment is a difficult problem (Ager et al. 2011), and the question of how to carry out the rapid assessment and update of grassland fire risk has become a hot issue in grassland fire research.

Grassland fire risk assessment methods include the grassland fire risk probability method and the grassland fire risk index method. Probabilistic risk assessment (PRA) is a systematic and comprehensive methodology to evaluate the risks associated with a complex engineered technological 
entity or the effects of stressors on the environment (Goussen et al., 2016). Generally, PRA is defined as the outcome of probability multiplying potential losses (Finney 2005). For grassland fires, there are two types of risk that need to be assessed: the risk of the occurrence of fire under various grassland management scenarios and the risk to the ecosystem as a result of the fire and/or as a consequence of the fire management practices (Fairbrother and Turnley 2005). The main issues of this kind of risk assessment are implemented by estimating the probability distributions based on a mass of statistical samples of historical data (Brillinger 2003; Liu et al. 2010, 2012; Cao et al. 2015). Fire probability index (Chuvieco 2003), fire occurrence (Martínez-Fernández et al. 2005), burn probability (Ziesler et al. 2013) and ignition risk (Yohay et al. 2009; Sow et al. 2013) have been typically used to describe grassland fire risk. Because of the small sample sizes of grassland fire, fire probability is usually substituted with frequency. To address the problem of small sample sizes, several methods have been provided to calculate risk probability, such as the Monte Carlo, information diffusion, logistic regression, and weights of evidence models (Yohay et al. 2009; Cui et al. 2010; Liu et al. 2010; Zhang et al. 2010; Shen et al. 2012).

Grassland fire risk index methods include the single index method and the composite index method. The single index method uses one important factor that affects the occurrence of grassland fires, such as the moisture of grassland fuel or the drought index, to predict the occurrence of grassland fires. Several studies have developed the fuel dryness index, the fine fuel moisture index, and the fire weather index to analyse grassland fire danger (Keetch and Byram 1968; Snyder et al. 2006; Van Wagner 1987). The grassland risk comprehensive index predicts the possibility of a grassland fire by integrating various factors that affect the occurrence of grassland fires. These composite indices include the meteorological fire danger index, the fuel moisture index and the composite index, which 
include human activities and meteorological, topographical and fuel characteristics. These assessment results could help determine which aspects influence grassland fire risk. In the last few decades, because of the ease, convenience and rapid acquisition of data, remote sensing and geographic information system technology has been widely used in risk indices to improve the forecasting and monitoring of fire (Paltridge and Barber 1988; Jaiswal et al. 2002; Castro et al. 2003; Mbow et al. 2004; Hernandez-Leal et al. 2006; Gitas et al. 2014).

In the study of grassland fire, several methods and models have been applied to risk research, e.g., the information diffusion method, the fuzzy inference model and the machine learning model. Such methods and models are effective for analysis and evaluation within one region. However, when they are applied in different regions, due to the different distribution characteristics of vegetation, climate and human activities data in different regions, it will result in the deviation of assessment results. In traditional machine learning, two basic assumptions are needed in order to ensure the classification accuracy and reliability of training. (1) The training samples used for learning and the new test samples satisfy the independent and identically distributed conditions. (2) There must be sufficient available training samples to learn to develop a good model. In natural disaster studies, for areas lacking data, these two conditions are often difficult to satisfy. Therefore, a trained model in one region cannot be directly used in another region, and a parameter adjustment is always required (for remote areas, the parameter adjustment is often limited because of the lack of data).

At present, grassland fire risk has been widely studied around the world, and much experiential knowledge has been summarized. How to transfer this experiential knowledge to specific areas is an important issue for grassland fire studies. Another problem is that risk maps often need to be updated over time for grassland fire risk management, and the production of risk maps often requires many 
material resources and is time-consuming and labour-intensive. For areas lacking data, grassland fire risk evaluation results often cannot be obtained. To solve this problem, in this study, a new grassland fire risk assessment method was proposed based on transfer learning. Two major grassland areas (Xilingol and Hulunbuir) in northern China are selected as the study areas, and five representative indicators (features) are extracted from grassland fuel, fire climate, accessibility, and human and social factors. Taking Xilingol as the source domain (where sufficient labelled data are available) and Hulunbuir as the target domain (which contains insufficient data but requires risk assessment/prediction), we then establish the mapping relationship between grassland fire indicators and grassland fire risk degrees by using a transfer learning method. The fire risk was assessed in the Hulunbuir grassland based on the transfer learning method. This method could conveniently be used for risk mapping and updating, especially for the risk assessment of grassland fire in remote areas.

There are two major contributions of this study. 1) It proposes a new grassland fire risk mapping and updating method based on transfer learning. In this method, five representative indicators were extracted from fuel, fire climate, accessibility, and human and social factors. Considering the lack of data in remote areas, the remote-sensing data were used to obtain fuel and road networks in the study area. 2) The study addresses the issue of transferring grassland fire risk knowledge and experience from one region to another. As a result, this study will allow for the transfer of knowledge and experience from well-studied regions of grassland fire risk to poorly studied regions, which will reduce grassland fire risk management costs. This study can also be used to update grassland fire risk maps and perform grassland fire risk parameter optimization.

\section{Grassland fire risk analysis method based on transfer learning}


111

existing grassland fire risk research. According to grassland fire risk literature (Cardille et al. 2001;

Castro et al. 2003; Hernandez-Leal et al. 2006; Marta et al. 2008; Cui et al. 2010; Chuvieco et al. 2014), grassland fire risk is affected by multiple factors. Grassland fire risk factors can be classified as ignition factors, fuel factors, meteorological factors, and fire impacts. Fuel is the basis of fire propagation. Weather conditions determine fuel moisture and further determine flammability. The sources of ignition can be divided into human-caused and lightning-caused fires. Several studies have shown that more than $90 \%$ of grassland fires were caused by humans (Liu et al. 2012; Zhang et al. 2015); therefore, this study uses accessibility to describe fires caused by humans. The impacts of fire include both ecological and economic losses. This study uses population density and economic density to describe fire impacts. The combination of the four considered factors (five indicators) has caused different fire risks in grasslands. In this study, the factors in the study area were obtained and processed as input factors to predict grassland fire risk based on the transfer learning method.

\subsection{Fuel}

Fuel is a critical element for the formation and spread of grassland fires. In a combustion science context, fuels are any combustible material (NWCG 2006). In a grassland, these combustible materials are the live and dead grass that ecologists call biomass (Keane 2015). Grassland areas with abundant fuel tend to be prone to fire. Therefore, the knowledge of the spatial distribution of these fuels is essential to developing fire management strategies. For large-scale spatial grassland areas, grassland fuel characteristics can be obtained from remote sensing images. At present, several remote-sensing techniques have been developed to map fuels at different resolutions on the Earth's surface (Arroyo et al. 2008). Several studies have proven the feasibility of assessing fire risk by using a vegetation index such as the NDVI (Mbow et al. 2004; Marta et al. 2008), but the limitations of the NDVI itself may 
133

affect the obtained grassland load estimates. Specifically, in low cover grassland areas, the estimated results exhibit high error rates because of the significant influences of the soil background and grassland vegetation types. Net primary productivity (NPP) is defined as the total photosynthetic gain, minus respiratory losses, of vegetation per unit ground area (Scurlock et al. 2002). NPP measures the cumulative amount of carbon elements in the plants per unit area per unit time interval. The mass of carbon per unit area per year $\left(\mathrm{g} \mathrm{C} \mathrm{m}^{-2} \mathrm{yr}^{-1}\right)$ is most often used as the unit of measurement. Therefore, it is very suitable for measuring the fuel load in a grassland. Several studies have proven that NPP could measure grass load in the grassland (Wang et al. 2012; Zhao et al. 2014; Ni 2004). Because fuel combustion is related to carbon elements, in this study, NPP is used to calculate the fuel load in the grassland. The MOD17 product is a land productivity product calculated using the BIOME-BGC model and a light use efficiency model, in combination with remote-sensing data. In this study, the yearly product MOD17A3 data from 2000 to 2014 were used to analyse the fuel load in the grassland.

$\mathrm{FI}=\frac{1}{n} \sum_{i=1}^{n} N P P_{i}$

where $F I$ is the fuel load index $\left(\mathrm{g} \mathrm{C} \mathrm{m}^{-2}\right), N P P_{i}$ is the net primary productivity in the $i$ th year, and $n$ is the total number of years.

\subsection{Fire climate}

The ignition and propagation of grassland fires are also influenced by local weather conditions (Bian et al. 2013). The fire climate affects the fuel moisture (Snyder et al. 2006) and then affects the intensity of the grassland fire. Such climate also influences the spread speed of a grassland fire. Well-known integrated indices of fire climate include the Canadian Forest Fire Weather Index (CFFWI) (Dowdy et al. 2009), the Keetch-Byram Drought Index (KBDI) (Keetch and Byram 1968) and the Fire Danger Index (FDI) (Pitman et al. 2007). The CFFWI is a numerical rating of fire intensity, which is 
155

156

157 the comprehensive fire weather danger index.

172

Table 1 Single meteorological factor lookup table

\begin{tabular}{llllllll}
\hline $\boldsymbol{v}(\mathrm{m} \mathrm{s}-1)$ & $\boldsymbol{I}_{\boldsymbol{v}}$ & $\mathrm{T}\left({ }^{\circ} \mathrm{C}\right)$ & $\boldsymbol{I}_{\boldsymbol{T}}$ & $\mathrm{F}(\%)$ & $\boldsymbol{I}_{\boldsymbol{F}}$ & $\mathrm{NP}(\mathrm{d})$ & $\boldsymbol{I}_{\boldsymbol{m}}$ \\
\hline $0-0.9$ & 5 & $15-19$ & 0 & $>75$ & 0 & 0 & 0 \\
$1.0-2.9$ & 15 & $20-23$ & 3 & $40-75$ & 5 & 1 & 5 \\
$3.0-5.9$ & 25 & $24-28$ & 6 & $25-39$ & 10 & 2 & 10 \\
$6.0-10.9$ & 30 & $29-32$ & 9 & $15-24$ & 15 & $3-5$ & 15 \\
$\geq 11.0$ & 35 & $33-37$ & 12 & $8-14$ & 20 & $6-8$ & 20 \\
- & - & $>38$ & 15 & $0-7$ & 25 & $>8$ & 25 \\
\hline
\end{tabular}

where $I_{n m c}$ is the comprehensive fire weather danger index, $U$ is the fire weather danger index before adjustment, and $U^{\prime}$ is the adjusted fire weather danger index. $I(s)$ and $I^{\prime}(s)$ are the corresponding fire weather danger values for each single meteorological factor, and they could be obtained from the lookup tables (Tables 1 and 2). $v$ is the daily maximum wind speed ( $\left.\mathrm{m} \mathrm{s}^{-1}\right), T$ is the maximum temperature $\left({ }^{\circ} \mathrm{C}\right), r h$ is the minimum relative humidity $(\%)$, and $\mathrm{F}$ is the sum of fuel moisture and relative humidity multiplied by $0.25 . \mathrm{m}$ is continuous non-precipitation (NP) days. $\mathrm{A}=0.3$ and $\mathrm{B}=0.7 . C_{s}$ is the surface correction coefficient $[0,1] . C_{r}$ is the correction coefficient of precipitation; $C_{r}=0$ when there is precipitation, and $C_{r}=1$ when there is no precipitation. $I_{n m c}$ is 
Table 2 Adjusted meteorological factor lookup table

\begin{tabular}{cccccccc}
\hline $\boldsymbol{v}(\mathrm{m} \mathrm{s}-1)$ & $\boldsymbol{I}_{\boldsymbol{v}}$ & $\mathrm{T}\left({ }^{\circ} \mathrm{C}\right)$ & $\boldsymbol{I}_{\boldsymbol{T}}$ & $r h(\%)$ & $\boldsymbol{I}_{\boldsymbol{r h}}$ & $\mathrm{NP}(\mathrm{d})$ & $\boldsymbol{I}_{\boldsymbol{m}}$ \\
\hline $0-1.5$ & 3.846 & $\leq 5$ & 0 & $\geq 70$ & 0 & 0 & 0 \\
$1.6-3.4$ & 7.692 & $5-10$ & 4.61 & $60-70$ & 3.076 & 1 & 7.692 \\
$3.5-5.5$ & 11.538 & $11-15$ & 6.1 & $50-59$ & 6.153 & 2 & 11.538 \\
$5.6-8.0$ & 15.384 & $16-20$ & 9.23 & $40-49$ & 9.23 & 3 & 19.23 \\
$8.1-10.8$ & 19.236 & $21-25$ & 12.5 & $30-40$ & 12.307 & 4 & 23.076 \\
$10.9-13.9$ & 23.076 & $>25$ & 15.384 & $<30$ & 15.384 & 5 & 26.923 \\
$14.0-17.2$ & 26.923 & - & - & - & - & 6 & 30.7 \\
$>17.2$ & 30.9 & - & - & - & - & 7 & 34.615 \\
- & - & - & - & - & - & $>8$ & 38 \\
\hline
\end{tabular}

In this study, $C_{s}$ is used to measure the influence of aspect, which is calculated by the DEM of the belongs to a high-danger area if $I_{n m c}>60$. The frequency of the high fire weather danger index was

$$
a_{2}=1.6551+0.6625 x-0.0510 x^{2}
$$

where $x$ is the air temperature.

Based on Eqs. 2-7, the daily fire weather danger index values were calculated in the research area. According to the standards for the grade classification of the fire weather danger index, a region

$$
F C I=\frac{N}{M}
$$


190

191

192

193

194

195

196

197

198

199

200

201

202

203

204

205

206

207

where FCI is the frequency of the high fire weather danger index in the region (\%), $N$ is the $I_{n m c}>$ 60 days in the reported years, and $M$ is the total days in the reported years.

\subsection{Accessibility}

The vast majority of contemporary wildfire ignitions globally are of human origin, and several studies have examined the impact of socio-economic and human activities on grassland fire risk (Martínez et al. 2009; Cardille et al. 2001). Factors related to the social economy and human activities, such as agricultural area, density of roads, population, etc., were used to establish the grassland fire risk.

Human-caused fires are closely related to the range of human activities. In this study, accessibility was used to express the scope and intensity of human activities. Human activities and habitats/settlements are always distributed along roads. Human, animal and vehicular movement and activities such as cooking, camping and smoking on roads provide ample opportunities for accidental/man-made fires.

Studies have often found roads to be related to accidental or negligent fire occurrence (Cardille et al. 2001). Therefore, grasslands near roads and habitats appear to be at high fire risk (Jaiswal et al. 2002). Since habitats/settlements are generally close to the road network, this study mainly considers the impact of road networks on the grassland fire risk. In this study, accessibility was used to express the degree of fire danger caused by human activities and was understood to be the distance from roads. Grassland areas near roads have high accessibility scores, and these areas are more prone to ignition via human activities or vehicle movement. As in previous studies (Jaiswal et al. 2003; Bian et al. 2013), the indicator 'distance to roads' was divided into five grades and assigned values as shown in Table 3.

\subsection{Human and social factors}

Grassland fire has a severe influence on local residents and the social economy. In China, grassland fire is regarded as a serious natural disaster that affects the development of grassland areas. It has 
Table 3 Rating values and classes assigned to factors for grassland fire risk.

\begin{tabular}{|c|c|c|c|}
\hline Primary factors & Secondary factors & Classes & Rating values \\
\hline Accessibility & Distance to roads (DTR, m) & $\begin{array}{l}0-1000 \\
1000-2000 \\
2000-3000 \\
3000-4000 \\
>4000\end{array}$ & $\begin{array}{l}1.0 \\
0.8 \\
0.6 \\
0.4 \\
0.2\end{array}$ \\
\hline \multirow{2}{*}{$\begin{array}{l}\text { Human and } \\
\text { social factors }\end{array}$} & $\begin{array}{l}\text { Population density } \\
\left(\mathrm{PD}, \text { person } / \mathrm{km}^{2}\right)\end{array}$ & $\begin{array}{l}0-10 \\
10-100 \\
100-1000 \\
1000-2000 \\
>2000\end{array}$ & $\begin{array}{l}0.2 \\
0.4 \\
0.6 \\
0.8 \\
1.0\end{array}$ \\
\hline & $\begin{array}{l}\text { Animal husbandry output } \\
\left(\mathrm{AHO}, 10^{4} \mathrm{CNY} / \mathrm{km}^{2}\right)\end{array}$ & $\begin{array}{l}0-10 \\
10-100 \\
100-500 \\
500-1000 \\
>1000\end{array}$ & $\begin{array}{l}0.2 \\
0.4 \\
0.6 \\
0.8 \\
1.0\end{array}$ \\
\hline
\end{tabular}
previously acquired knowledge to solve new but similar problems much more quickly and effectively.

burned out pastures and caused livestock to starve to death, or burned them directly, which can destroy the local economy. For residents, grassland fires can also burn down their houses and destroy living supplies and even affect their lives and safety. In this study, population density and animal husbandry output are used to measure the impact of grassland fire on population and society. Grassland areas with high population density and animal husbandry output have high risk of grassland fires. The two indicators 'population density' and 'animal husbandry output' were divided up into five grades and assigned values, as shown in the Table 3.

\subsection{Transfer learning based on domain adaptation}

Transfer learning, as a new machine learning method, aims to provide a framework to utilize It has emerged in the computer science literature as a means of transferring knowledge from a source domain to a target domain ( $\mathrm{Lu}$ et al. 2015). The definition of transfer learning was proposed by Pan et 
al. 2010. First, the domain was defined as $D=\{\chi, P(X)$, which consists of two components: feature space $\chi$ and marginal probability distribution $P(X)$, where $X=\left\{x_{1}, x_{2}, x_{3}, \cdots, x_{n}\right\} \in \chi$. Next, the task was defined as $T=\{Y, f(\cdot)\}$, which consists of a label space $Y=\left\{y_{1}, y_{2}, y_{3}, \cdots, y_{m}\right\}$ and an objective predictive function $f(\cdot)$, which is not observed and is to be learned by pairs $\left\{x_{i}, y_{i}\right\}$. Finally, by giving the source domain $D_{s}$, target domain $D_{t}$, learning task $T_{s}$ and $T_{t}$, the purpose of transfer learning is to improve the learning of the target predictive function $f_{t}(\cdot)$ in $D_{t}$ using the knowledge in $D_{s}$ and Ts $\left(D_{s} \neq D_{t}\right.$ or $\left.T_{s} \neq T_{t}\right)$. Transfer learning uses the labelled source domain data to learn the calibration of the target domain data. The task of transfer learning is how to use labelled source domain data to establish a reliable model to predict the data in the target area (the source data and the target data have different probability distributions).

Transfer learning can be used to transfer the research experience of one natural disaster to another, and it is also possible to transfer the knowledge and experience of natural disaster research within one region to another. In general, source domains can differ in some combination of (often unknown) factors, including fire climate, fuel characteristics, and human and societal factors. To address this problem, domain adaptation algorithms are used to transfer knowledge from source domain trained on some available labelled data to the target domain. Therefore, domain adaptation solves a learning problem in a target domain by utilizing the training data in a different but related source domain (Pan et al. 2010). In grassland fire risk analysis, we do not know whether the selected sample in the target domain is representative; therefore, in this study, a feature-based domain adaptation method, transfer component analysis (TCA), which was proposed by Pan et al. (2011), was used to analyse the grassland fire risk. This method assumes that some labelled data are available in the source domain and that only unlabelled data are available in the target domain. The calculation steps are as follows: 
(1) Input unlabelled dataset in the target domain, $D_{t u}=\left\{x_{\text {tar1 } 1}^{u}, x_{\text {tar2 }}^{u}, \cdots, x_{\text {tarm }}^{u}\right\}$, unlabelled dataset in the source domain, $D_{s u}=\left\{x_{s r c 1}^{u}, x_{s r c 2}^{u}, \cdots, x_{s r c n}^{u}\right\}$, and selecting the labelled dataset in the source domain, $D_{s l}=\left\{x_{s r c 1}^{l}, x_{s r c 2}^{l}, \cdots, x_{s r c n}^{l}\right\}$.

(2) Calculate the distance between the two domains. The maximum mean discrepancy (Borgwardt et al. 2006) was used to calculate the distance between the source domain and the target domain.

$$
\operatorname{dis}\left(X_{s r c}^{\prime}, X_{t a r}^{\prime}\right)=\left\|\frac{1}{n_{1}} \sum_{i=1}^{n_{1}} \varnothing\left(x_{s r c_{i}}\right)-\frac{1}{n_{2}} \sum_{i=1}^{n_{2}} \varnothing\left(x_{t a r_{i}}\right)\right\|_{\mathcal{H}}^{2}
$$

where $\mathcal{H}$ is a reproducing kernel Hilbert space (Steinwart, 2001), and $\emptyset: x \in \chi \rightarrow \mathcal{H}$. According to a

$$
k\left(x_{i}, x_{j}\right)=\varnothing\left(x_{i}\right)^{\prime} \emptyset\left(x_{j}\right)
$$

where $\mathrm{k}$ is the corresponding kernel. Therefore, the distance between the source domain and the target

$$
\operatorname{dis}\left(X_{s r c}^{\prime}, X_{t a r}^{\prime}\right)=\operatorname{tr}(K L)
$$

where

$$
K=\left[\begin{array}{ll}
K_{S, S} & K_{S, T} \\
K_{T, S} & K_{T, T}
\end{array}\right]
$$
calculated as follows.

$$
L_{i j}=\left\{\begin{array}{cc}
\frac{1}{n_{1}^{2}} & x_{i}, x_{j} \in X_{s r c}, \\
\frac{1}{n_{2}^{2}} & x_{i}, x_{j} \in X_{t a r}, \\
-\frac{1}{n_{1} n_{2}} & \text { otherwise }
\end{array}\right.
$$




$$
\widetilde{K}=\left(K K^{-1 / 2} \widetilde{W}\right)\left(\widetilde{W}^{T} K^{-1 / 2} K\right)=K W W^{T} K
$$

$$
W=K^{-1 / 2} \widetilde{W}
$$

$$
\operatorname{dis}\left(X_{\text {src }}^{\prime}, X_{\text {tar }}^{\prime}\right)=\operatorname{tr}\left(\left(K W W^{T} K\right) L\right)=\operatorname{tr}\left(W^{T} K L K W\right)
$$

$$
\min _{W} \operatorname{tr}\left(W^{T} K L K W\right)+\mu \operatorname{tr}\left(W^{T} W\right)
$$

$$
\text { s.t. } W^{T} K H K W=I_{m}
$$

where $H$ is the centering matrix, and 1 is a column vector with all ones.

(3) By calculating the distance between two domains, the features of the two domains are prediction model trained for the source domain can also be used to solve the tasks in the target domain. 
291 where $P$ is the probability that an input $(x)$ belongs to the default class $(\hat{y}=1,2,3)$, and $\beta_{\mathrm{i}}$ is a

292 coefficient. To improve classification accuracy, the predicted classification is calculated to minimize

293 the expected classification loss:

295 where $\hat{y}$ is the predicted classification. $\mathrm{D}$ is the number of classes. $\hat{P}(d \mid x)$ is the posterior probability of class $d$ for observation $x . C(y \mid d)$ is the loss of classifying an observation as $y$ when its true

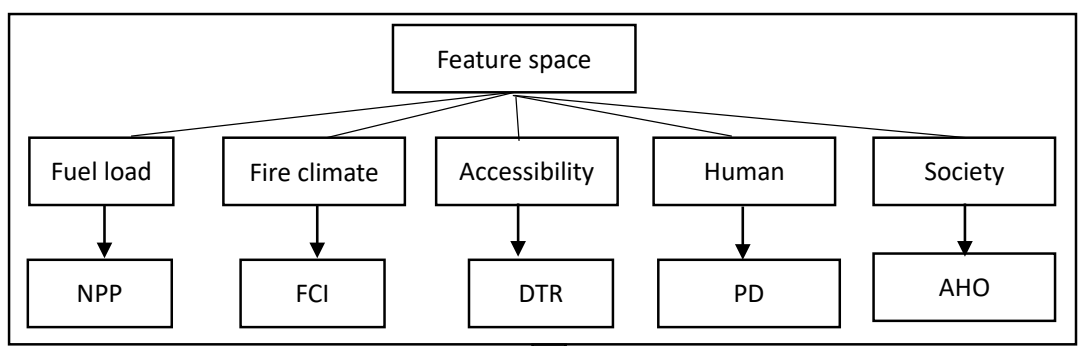

$5 \zeta$

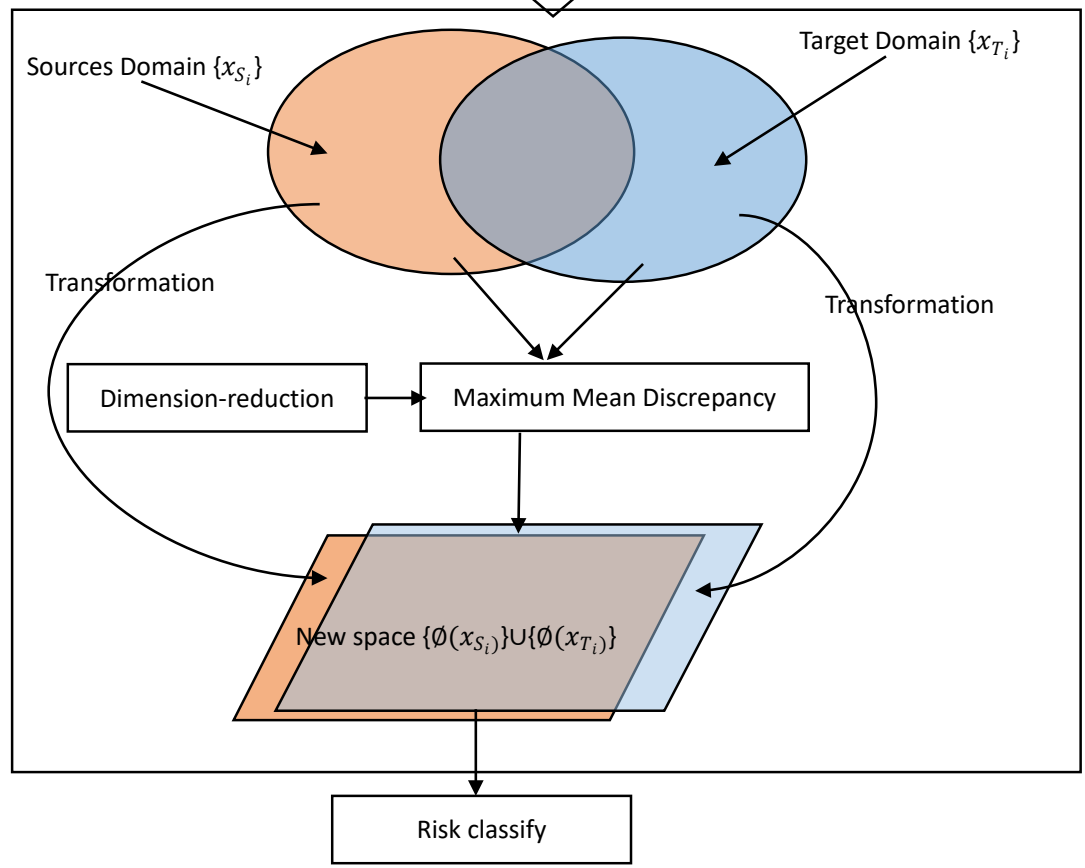

Fig. 1. Analysis flowchart of grassland risk based on transfer learning 
Transfer learning addresses the problem of how to leverage previously acquired knowledge to improve the efficiency and accuracy of learning in another domain that in some way and to some extent relates to the original domain (Pan and Yang 2010). Such learning represents the ability of a system to apply the knowledge of previous tasks to a new domain or new tasks. Traditional machine learning algorithms operate under the hypothesis that training data (source domain) and the test data (target domain) have identical feature spaces with the same underlying distribution. Unlike traditional algorithms, transfer learning considers that the domains of the training data and the test data may be different (Daume and Marcu 2006, Fung et al. 2006). The transfer learning model is more feasible than the traditional mathematical model, and the analysis results are more reliable. There is a more effective use of available data to improve the generalization of the model to make the model more robust, and it is a good tool for model parameter adjustment. Therefore, in this study, feature-based transfer learning methods were used to analyse the grassland fire risk in different regions.

Although transfer learning methods can transfer and incorporate knowledge and experience from different regions, we need to select robust features to reduce the difference between the source and target domains and to reduce fire risk assessment errors. Because some indicators have conflicting knowledge and experience of grassland fires in different regions (e.g., the composition of fuels and the month of fire occurrence vary greatly in Asia, Africa and Australia), the selection of such indicators may lead to negative transfer. If we want to assess the grassland fire risk in one grassland using the knowledge and experience of another grassland based on transfer learning, it is better to select a grassland with similar knowledge and experience or select robust indicators (e.g., indicators that are similar between regions) in order to reduce the assessment error.

\section{Real-world applications and result analysis}


properties of our framework. These study areas are two major grasslands in northern China that are mean NPP is $25.36 \mathrm{~g} \mathrm{C} \mathrm{m}^{-2}$, and the variance is $10.76 \mathrm{~g} \mathrm{C} \mathrm{m}^{-2}$. According to the meteorological data,

a
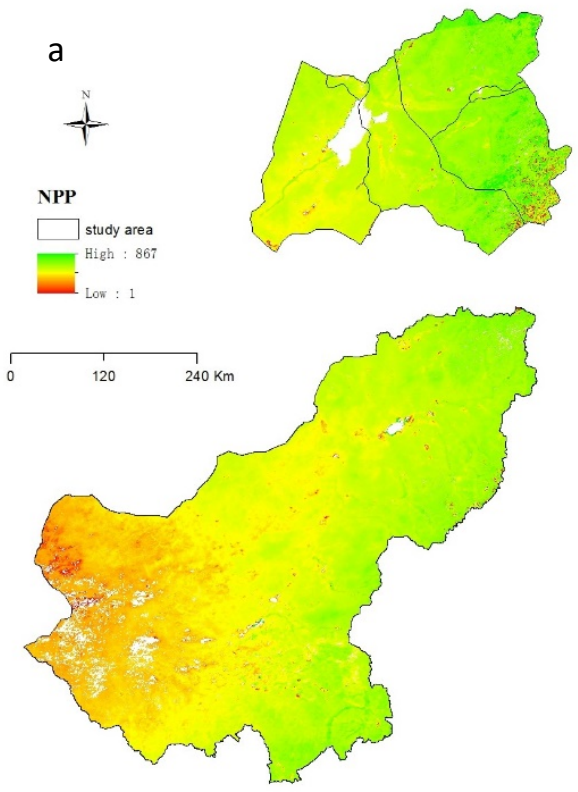

b
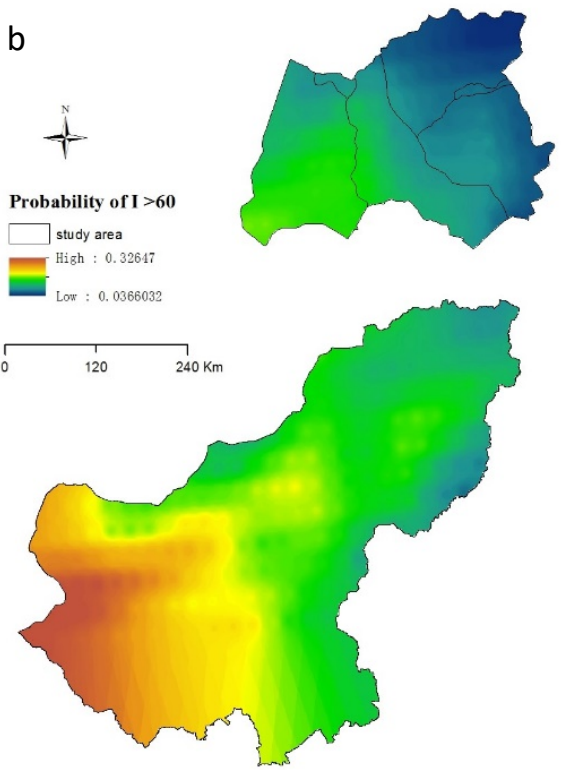

Fig. 2. The spatial distribution of NPP (a) and the frequency of high grassland fire danger weather 
index $\left(I_{n m c}>60\right)(\mathrm{b})$ in the study areas.

The comparison of the fuel load index and the fire weather danger index of the two grassland areas shows that they are very different (Fig. 3). The Hulunbuir grassland has better vegetation and less severe fire weather than the Xilingol grassland. The grassland fire risk situation in Xilingol is worse

Xilingol. Therefore, the transfer learning method was applied to these two grassland areas to verify the experience of grassland fire risk and small labelled samples.
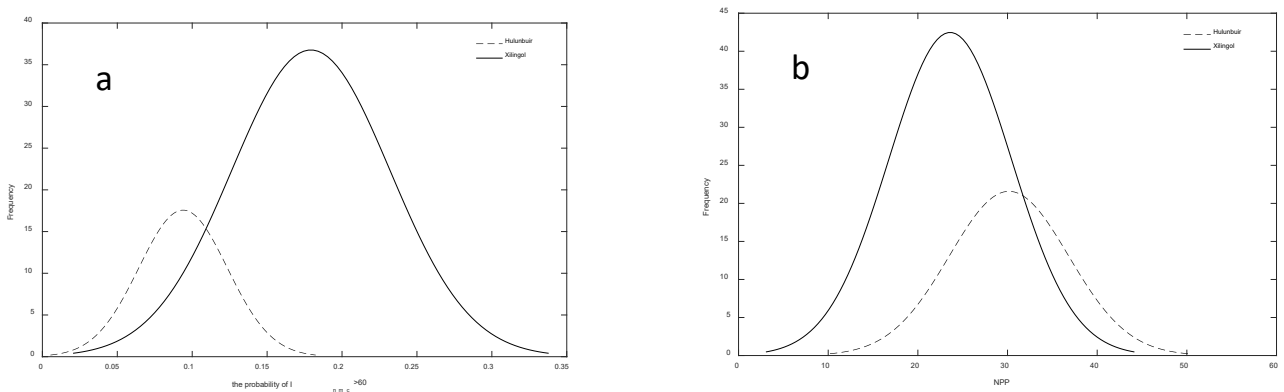

Fig. 3. The frequency distributions of $I_{n m c}>60$ (a) and NPP (b) in two grassland areas.

In this study, the fire risk map of the Xilingol grassland is regarded as the source domain. The are shown in Fig. 5. In this risk map, 500 random selected samples were sampled on the source domain risk maps, which were used to train samples (Fig. 4(a)), and the percentages of high-, medium- and low-risk samples were $40 \%, 38 \%$ and $22 \%$, respectively. The Hulunbuir grassland was selected as the 

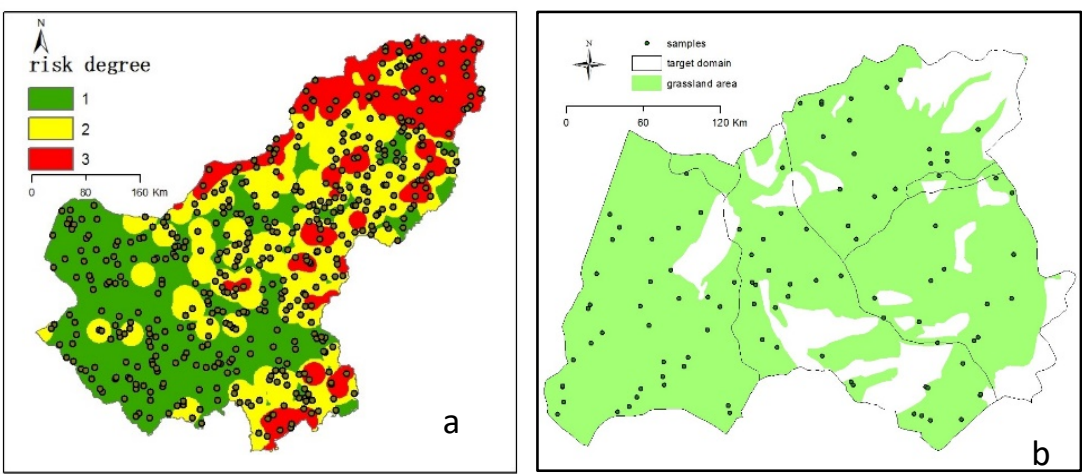

Fig. 4. The spatial distribution of grassland fire risk and selected samples in Xilingol (a), and the

grassland area and selected samples in Hulunbuir (b). 1, 2, and 3 represent low, medium, and high risk,

respectively.

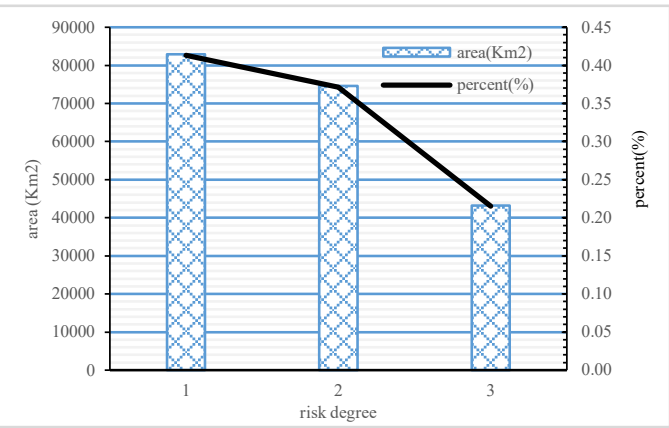

Fig. 5. The ratio and area of risk degrees in Xilingol

In this study, the traditional dimensionality reduction method of principal component analysis

(PCA) was used to project the original data to a low-dimensional latent space while preserving some of

the properties of the original data (Fig. 6). Analysing the labelled samples revealed that the contribution to analyse the risk of grassland fire. 


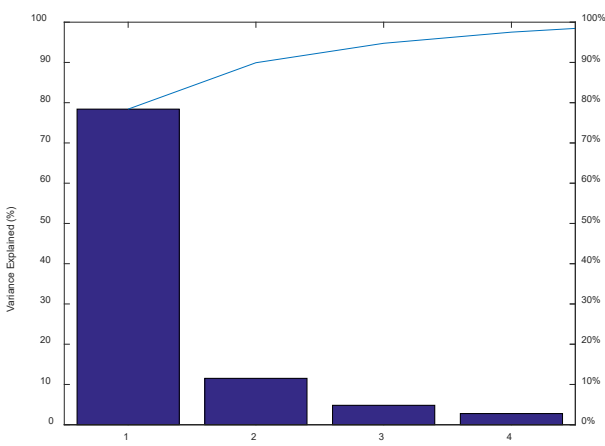

Fig. 6. The principal components of grassland fire risk

Based on the selected factors and transfer learning method proposed in this study, the spatial areas in the middle areas are dispersed. The risk of grassland fire in the northern part of the Hulunbuir grassland is higher than that in the southern region (Fig. 7).

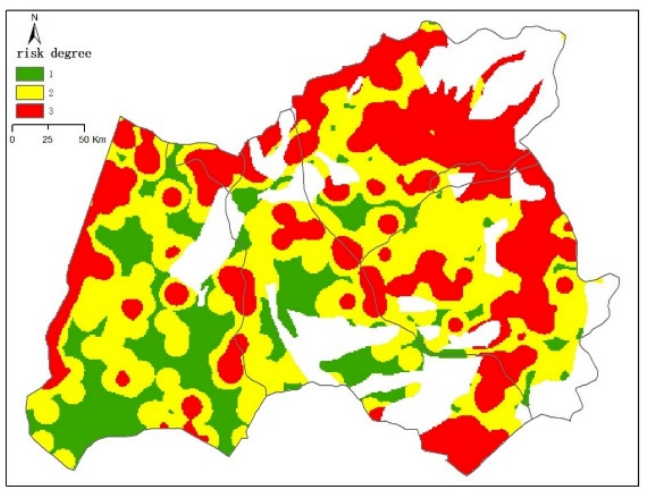

Fig. 7. The results of grassland fire risk assessment of Hulunbuir based on transfer learning

Natural fire rotation (NFR) (Heinselman, 1973) was used to verify the risk results of the Hulunbuir grassland fire in this study. Assuming that the landscape is uniform, and the burning conditions are constant over time (i.e., ignition frequency, and climatology), the NFR reflects the time required to burn an area equal in size to the study area. The NFR is calculated as:

$$
N F R=A_{t}\left(A_{f} / N_{y}\right)
$$


384

385

386

387

388

389

390

391

392

393

394

395

396

397

where $A_{t}$ is the total area of the land, $A_{f}$ is the total area burned by all fires (including re-burned areas) and $N_{y}$ is the number of years in the record. Accuracy, sensitivity, specificity, precision, recall, F-measure, and G-mean (Kubat et al. 1997) were selected as metrics to evaluate the goodness of assessment results. The comparison between the Hulunbuir grassland fire risk and NFR shows that transfer learning has high prediction accuracy for medium-risk areas and low-risk areas $(91 \%$ and $95 \%$, respectively) (Fig. 8 and Table 4), while the prediction accuracy rate for high-risk areas is low (67\%). Some high-risk areas have been predicted to be middle-risk areas.

From Fig. 8, it can be seen that the accuracy of low grassland fire risk assessment and middle grassland fire risk assessment is high, while the misreporting rate of high grassland fire is high (33\%). This is due to the real grassland fire risk being based on the occurrence of fire, while the grassland fire risk in this study was assessed based on five selected indicators. The 'distance to roads' indicator was chosen to describe the human activity in this study. For some isolated areas with medium fire risk, such as areas with high fuel load and far from roads and where human activities are primarily tourism, herbs collecting, etc., grassland fire risk is high due to natural fires and human activities.

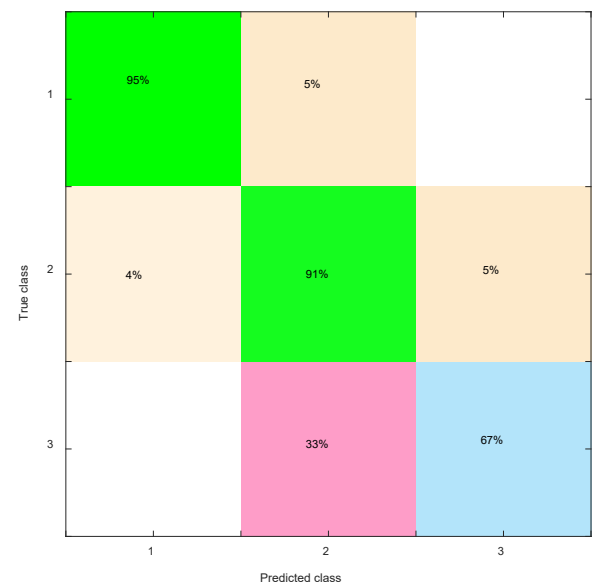

Fig. 8. The accuracy of grassland fire risk assessment based on transfer learning 


\begin{tabular}{|c|c|c|c|c|c|c|}
\hline accuracy & sensitivity & specificity & precision & recall & F-measure & G-mean \\
\hline 0.8746 & 0.9302 & 0.8662 & 0.9128 & 0.9302 & 0.6612 & 0.8976 \\
\hline
\end{tabular}
that the predicted reliability was satisfactory.

Because the number of samples has an important impact on the expression of features, this study analyses the impact of samples on the evaluation results (Fig. 9). In this study, we set the number of 


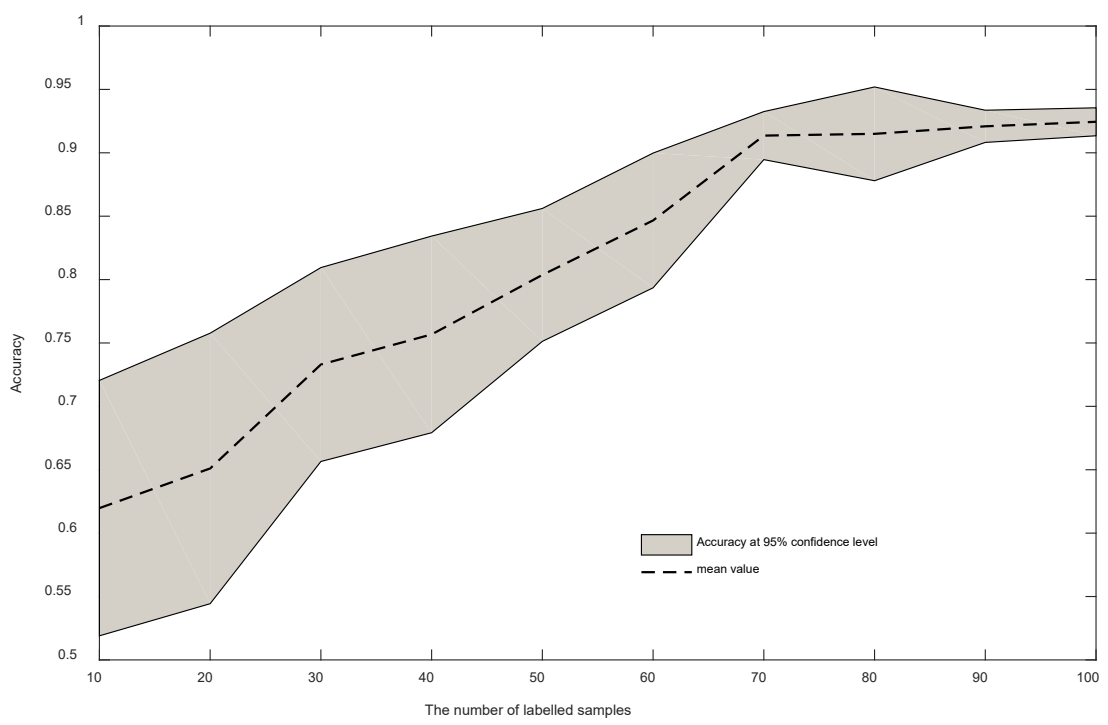

418 Fig. 9. The impact of the number of labelled samples on the evaluation results

419 Fig. 9 shows that the grassland fire risk was affected by the number of labelled samples and that the accuracy of assessment results increased with the number of labelled samples. The reason for this result experience embodied in the labelled samples will overlap, and their impact on the prediction accuracy will be stabilized.

\section{Conclusions and further study}

This study selected five indicators from grassland fuel, fire climate, accessibility, and human and social factors. By constructing the source domain and the target domain samples, the transfer learning method was used to construct the relationship between grassland fire risk factors and fire risk grades. This method verified the reliability of mapping grassland fire risk in different regions based on existing knowledge and experience. 
characteristics in different areas and then evaluate the grassland fire risk in different areas. In future studies, the grassland fire risk in unequal feature spaces (two study areas with fewer of the same factors) will be studied based on the transfer learning method.

This research revealed that the five selected indicators and the designed framework are reliable in grassland fire risk assessment, and they can be used for grassland fire risk assessment. The number of labelled samples has an impact on the accuracy of grassland fire risk mapping. Because randomly selected labelled samples were used in this study, the feature of grassland fire risk is easily affected by negative samples. In this study, 60 selected labelled samples were found to be the minimum required to meet the requirements. Because of the importance of information in samples, to ensure the accuracy of assessment results, we suggest that fire managers select independently labelled samples to increase learning knowledge and experience as much as possible.

\section{Acknowledgements}

This study is supported by the Fundamental Research Funds for the Central Universities (2412017FZ023), Jilin Provincial Department of Education (JJKH20190285KJ), the National Natural Science Foundation of China under Grant No. 41071326, and the China Scholarship Council (201706625009)

\section{References}

Ager, A. A., Vaillant, N. M., \& Finney, M. A., 2011. Integrating fire behaviour models and geospatial analysis for wildland fire risk assessment and fuel management planning. Journal of Combustion 2011, 19 pages.

Arroyo, L. A., Pascual, C., Manzanera, J. A., 2008. Fire models and methods to map fuel types: the role of remote sensing. Forest Ecology \& Management 256(6), 1239-1252. 
Cao, X., Meng, Y., Chen, J., 2015. Mapping Grassland Wildfire Risk of the World. World Atlas of in the Upper Midwest. United States Ecological Applications 11, 111-127.

Castro F.X., Tudela A., Sebastià M.T., 2003. Modeling moisture content in shrubs to predict fire risk in

Catalonia (Spain). Agricultural and Forest Meteorology 116, 49-59.

473 World Scientific Publishing Co. Pty Ltd: Singapore.

474 Chuvieco, E., Aguado, I., Jurdao, S., Pettinari, M. L., Yebra, M., \& Salas, J., et al., 2014. Integrating 
477

478

479

480

481

482

483

484

485

486

487

488

489

490

491

492

493

494

495

496

497

498

Cui, L., Zhang, J. Q., Liu, X. P., Tong, Z. J., \& Kun-Peng, Y. I., 2010. Logistic regression-based prairie fire hazard prediction in case of Hulunbeier grassland. Journal of Safety \& Environment 10(1), $173-177$.

Daume, H., III; Marcu, D., 2006. Domain adaptation for statistical classifiers. Journal of Artificial Intelligence Research 26(1), 101-126.

Dowdy, A. J., Mills, G. A., Finkele, K., de Groot, William, 2009. Index sensitivity analysis applied to the Canadian Forest Fire Weather Index and the McArthur Forest Fire Danger Index. Meteorological Applications 17 (3): 298-312. doi:10.1002/met.170.

Fairbrother A., Turnley J. G., 2005. Predicting risks of uncharacteristic wildfires: Application of the risk assessment process. Forest Ecology and Management 211, 28-35.

Finney M. A., 2005. The challenge of quantitative risk analysis for wildland fire. Forest Ecology and Management 211, 97-108.

Fung G.P.C., et al., 2006. Text classification without negative examples revisit. IEEE Trans. Knowl. Data Eng. 18 (1), 6-20.

Gitas, I. Z., San-Miguel-Ayanz, J., Chuvieco, E., \& Camia, A., 2014. Advances in remote sensing and GIS applications in support of forest fire management. International Journal of Wildland Fire 23(5), 603-605.

Goussen B., et al., 2016. Integrated presentation of ecological risk from multiple stressors. Scientific Reports. 6. doi:10.1038/srep36004. ISSN 2045-2322. PMC 5080554 Freely accessible. PMID 27782171.

Heinselman, M.L., 1973. Fire in the virgin forests of the Boundary Waters Canoe Area, Minnesota. Quarter. Res. 3, 329-382. 
Hernandez-Leal, P. A., Arbelo, M., \& Gonzalez-Calvo, A., 2006. Fire risk assessment using satellite data. Advances in Space Research 37(4), 741-746.

Jaiswal, R. K., Mukherjee, S., Raju, K. D., Saxena, R., 2003. Forest fire risk zone mapping from satellite imagery and GIS. International Journal of Applied Earth Observation \& Geoinformation 4(1), $1-10$.

Kandya, A.K., Kimothi, M.M., Jadhav, R.N., Agarwal, J.P., 1998. Application of GIS in identification of fire prone areas-a feasibility study in parts of Junagarh (Gujrat, India). Indian For. 124 (7), 531-535.

Keane, R. E., 2015. Wildland Fuel Fundamentals and Applications. Springer International Publishing.

Keetch, J.J., Byram, G., 1968. A drought index for forest fire control. Res. Paper SE-38. U.S. Department of Agriculture, Forest Service, Southeastern Forest Experiment Station, Asheville, NC, 32 pp.

Kubat, M., Robert, and Matwin S., 1997. When negative examples abound. In Proceedings of the 9th European Conference on Machine Learning, ECML '97, pages 146-153, London, UK, Springer-Verlag.

Liu, X. P., Zhang, J. Q., Tong, Z. J., \& Bao, Y., 2012. GIS-based multi-dimensional risk assessment of the grassland fire in northern china. Natural Hazards 64(1), 381-395.

Liu, X., Zhang, J., Cai, W., \& Bao, Y., 2015. Estimating the insurance rates for loss of annual production of grass herbage associated with natural disasters in china. Rangeland Journal 37(2), 139-146.

Liu, X., Zhang, J., Cai, W., \& Tong, Z., 2010. Information diffusion-based spatio-temporal risk analysis of grassland fire disaster in northern china. Knowledge-Based Systems 23(1), 53-60.

Liu, Y. Q., Stanturf, J., Goodrick, S., Parks, C. G., Bernier, P., Bytnerowicz, A., et al., 2010. Trends in 
521 global wildfire potential in a changing climate. Forest Ecology \& Management 259(4), 685-697.

522 Lu, J., Behbood, V., Hao, P., Zuo, H., Xue, S., \& Zhang, G., 2015. Transfer learning using

523 computational intelligence: a survey. Knowledge-Based Systems 80(C), 14-23.

524 Marta, Y., Emilio, C., \& David, R., 2008. Estimation of live fuel moisture content from modis images

525 for fire risk assessment. Agricultural \& Forest Meteorology 148(4), 523-536.

526 Martínez, J., Vegagarcia, C., \& Chuvieco, E., 2009. Human-caused wildfire risk rating for prevention

527 planning in Spain. Journal of Environmental Management 90(2), 1241-1252.

528 Martínez-Fernández, J., Koutsias, N., Chuvieco, E., \& Allgöwer, B., 2005. Modelling Wildland Fire

529 Occurrence in Southern Europe by Geographically Weighted Regression Approach. 5th International

530 Workshop on Remote Sensing and GIS Applications to Forest Fire Management: Fire Effects

531 Assessment.

532 Mbow, C., Goita, K., Benie, G. B., 2004. Spectral indices and fire behavior simulation for fire risk 533 assessment in savanna ecosystems. Remote Sensing of Environment 91, 1-13.

534 Nelson, R., 1984. A method for describing equilibrium moisture content. Can. J. For. Res. 14, 597-600.

$535 \mathrm{Ni}$, J., 2004. Estimating net primary productivity of grasslands from field biomass measurements in 536 temperate northern china. Plant Ecology, 174(2), 217-234.

537 Niu. R., Zhi P., Sun M., 2006. Review of forest fire danger weather indexes and their calculation 538 methods. Meteorogical Monthly 32(12), 3-9.(Chinese)

539 NWCG, 2006. National Wildfire Coordination Group Glossary of Fire Terminology. http://www.

540 nwcg.gov/pms/pubs/glossary/.

541 Paltridge, G. W., \& Barber, J., 1988. Monitoring grassland dryness and fire potential in Australia with

542 NOAA/AVHRR data. Remote Sensing of Environment 25(3), 381-394. 
545 Pan, S.J. and Yang, Q. 2010. A survey on transfer learning. IEEE Trans. Knowl. Data Eng. 22 (10),

Pitman, A. J., Narisma, G. T., \& McAneney, J., 2007. The impact of climate change on the risk of forest and grassland fires in Australia. Climatic Change 84(3-4), 383-401. DOI: 10.1007/s10584-007-9243-6.

Scurlock, J.M.O.; Johnson, K.; Olson, R.J., 2002. Estimating net primary productivity from grassland doi:10.1046/j.1365-2486.2002.00512.x.

Snyder, R. L., Spano, D., Duce, P., Baldocchi, D., Xu, L., \& Paw U, K. T., 2006. A fuel dryness index

553 for grassland fire-danger assessment. Agricultural \& Forest Meteorology 139(1-2), 1-11. prescribed fire planning in Sudanian and Sahelian Savannas. Journal of Arid Environments 89(1), 84-93.

557 Steinwart, I., 2001. On the influence of the kernel on the consistency of support vector machines.

Thompson, M. P., Gilbertson-Day, J. W., \& Scott, J. H. 2015. Integrating pixel- and polygon-based approaches to wildfire risk assessment: application to a high-value watershed on the pike and San 
New York: Academic Press.

Wang, R. J. and Yang, L. W., 2012. Using RS Technology to Estimate Net Primary Production of Rangeland Ecosystem in Hulunbuir of China. Advanced Materials Research 365, 104-109.

Wang, Z., Song, Y., and Zhang, C., 2008. Transferred Dimensionality Reduction, Proc. European Conf. Machine Learning and Knowledge Discovery in Databases (ECML/PKDD ’08), pp. 550-565.

Yohay, C., Shlomit, P., Faris, J., \& Maxim, S., 2009. Assessing fire risk using Monte Carlo simulations of fire spread. Forest Ecology \& Management 257(1), 370-377.

Zadrozny B., 2004. Learning and Evaluating Classifiers under Sample Selection Bias, Proc. 21st Int'1 Conf. Machine Learning.

Zhang, J., Shen, L., Tong, Z., Liu, X., \& Cui, L., 2012. Spatial prediction of human-caused grassland fire risk in Hulunbeier region based on weights of evidence. Journal of Natural Disasters, 21(4), 99-107 (in Chinese).

Zhang, J.Q, Zhou, D.W., Wu, X.T. et al., 2006. A new perception on risk assessment and risk management of grassland fire disaster. J Basic Sci Eng (Supplement), pp 56-62 (in Chinese).

Zhang, Q., Cui, L., Zhang, J., Liu, X., \& Tong, Z., 2015. Grid based dynamic risk assessment for grassland fire disaster in hulunbuir. Stochastic Environmental Research \& Risk Assessment 29(2), 589-598.

Zhang, Z. X., Zhang, H. Y., \& Zhou, D. W., 2010. Using GIS spatial analysis and logistic regression to predict the probabilities of human-caused grassland fires. Journal of Arid Environments 74(3), 386-393.

Zhao, F., Xu, B., Yang, X., Jin, Y., Li, J., Xia, L., Chen, S., Ma H., 2014. Remote Sensing Estimates of Grassland Aboveground Biomass Based on MODIS Net Primary Productivity (NPP): A Case Study in 
590 Ziesler, P. S., Rideout, D. B., \& Reich, R., 2013. Modelling conditional burn probability patterns for

591 large wildland fires. International Journal of Wildland Fire 22(5), 579-587.

592

593

594 